\title{
ARTIGOS
}

\section{AVALIAÇÃO DA ESTABILIDADE NA CONTAGEM DE OVOS DE SCHISTOSOMA MANSONI PELO MÉTODO DE KATO-KATZ EM UMA ZONA ENDÊMICA DA ESQUISTOSSOMOSE *}

\author{
Maria Fernanda F. de Lima e Costa ${ }^{1}$, R.S. Rocha ${ }^{2}$ e Naftale Katz ${ }^{2}$
}

\begin{abstract}
A estabilidade da contagem de ovos de Schistosoma mansoni pelo método de KatoKatz foi avaliada em 144 pessoas residentes em uma área endêmica de Minas Gerais. Os resultados do primeiro exame foram comparados à média obtida em três exames de fezes realizados num periodo de 11 semanas. Os pacientes foram agrupados de acordo com a contagem de ovos obtida no primeiro exame de fezes: $<100,100-499,500-999 e \geqslant 1000$ ovos (classificação A); $<100,100-499 e \geqslant 500$ ovos (classificação B); $<500,500-999 e$ $\geqslant 1000$ ovos (classificação C). A percentagem de pacientes que permaneceram no mesmo grupo no qual haviam sido enquadrados no primeiro exame foi estatisticamente semelhante nas classificaçöes $A, B$ e C (73-81\%). O coeficiente de correlação de Pearson foi alto $(r=0,9038)$ e a média geométrica do número de ovos no primeiro exame $(575,7 \pm 5,4)$ foi semelhante a obtida em três exames de fezes $(556,6 \pm 4,4$ ovos/g fezes).
\end{abstract}

Palavras chaves: Esquitossomose mansoni. Schistosoma mansoni. Exame de fezes. Método de Kato-Katz.

O exame de fezes pelo método de Kato tem sido adotado para avaliar a "intensidade" da infecção pelo $S$. mansoni em diferentes áreas endêmicas da esquistossomose 1.34678101314 . Uma das questões que se colocam para o epidemiologista é se a contagem de ovos de $S$. mansoni obtida em um único exame é suficientemente estável para medir, de forma acurada, a eliminação de ovos do parasita nas fezes. De fato, trabalhos desenvolvidos no Brasil e no Quenia mostraram que o indice de estabilidade na contagem de ovos de $S$. mansoni pelo método Kato pode apresentar amplo limite de variação (cerca de 35 a 70\%) $)^{12} 1314$.

O presente trabalho foi desenvolvido em uma área endêmica situada no Estado de Minas Gerais com o objetivo de avaliar em que medida as contagens de ovos de $S$. mansoni obtidas em um e em très exames de fezes se correspondem.

\footnotetext{
* Trabalho desenvolvido no Centro de Pesquisas "René Rachou" - FIOCRUZ, financiado pelo Conselho Nacional de Desenvolvimento Científico e Tecnológico (CNPq) e pela Financiadora de Estudos e Projetos (FINEP).

1. Departamento de Medicina Preventiva e Social da Universidade Federal de Minas Gerais, Belo Horizonte.

2. Centro de Pesquisas "René Rachou" - FIOCRUZ, Belo Horizonte.

Recebido para publicação em 17/10/83.
}

\section{MATERIAL E MÉTODOS}

Em maio de 1981 foi feito um exame de fezes em $90 \%$ da população de Comercinho, cidade de 1474 habitantes, verificando-se que $70 \%$ dos pacientes examinados apresentavam ovos de $S$. mansoni nas fezes ${ }^{4}$.

Sessenta dias após a realização deste exame foi selecionada aleatoriamente uma amostra de 150 pessoas: 30 pacientes que apresentavam, respectivamente, 12 - 99, 100 - 499, 500 - 999, $1000-2999$ e $\geqslant 3000$ ovos de $S$. mansoni por grama de fezes no exame inicial. Nestes pacientes foram feitos mais dois exames em duas semanas consecutivas e os resultados obtidos no primeiro exame foram comparados à média obtida nos três exames de fezes.

Os três exames foram feitos pelo método de Kato modificado por Katz e cols ${ }^{9}$, preparando-se duas lâminas da amostra de fezes de cada paciente. A média do número de ovos de $S$. mansoni obtida em duas lâminas foi considerada como o número de ovos do paciente em cada exame de fezes. Todos os exames foram realizados pelo mesmo individuo e as laminas foram protocoladas de forma a não permitir que o examinador identificasse a origem do material.

Análise estatistica - $\mathrm{O}$ teste $t$ de Student foi usado para comparar a diferença entre a média geomé- 
Lima e Costa MFF, Rocha RS, Katz N. Avaliação da estabilidade na contagem de ovos de Schistosoma mansoni pelo método de Kato-Katz em uma zona endemica da esquistossomose. Revista da Sociedade Brasileira de Medicina Tropical 17: 7-12, JanMar, 1984

trica do número de ovos obtida no primeiro exame e a obtida nos très exames de fezes. O coeficiente de Pearson foi adotado para avaliar a correlação entre a contagem de ovos no primeiro exame e a média obtida em três exames de fezes de cada paciente. O teste do Qui quadrado. com correção de Yates, foi usado para comparar as diferenças entre as freqüèncias. $\mathrm{O}$ valor minimo de significancia considerado foi $95 \%(\mathrm{p}<0,05)^{15}$.

\section{RESULTADOS}

A estabilidade da contagem de ovos de $S$. mansoni foi avaliada em 144 pessoas, que constituiam
$96 \%$ dos pacientes selecionados para compor a amostra do presente trabalho.

A Tabela 1 mostra a comparação dos resultados obtidos em um e em três exames de fezes, quando os pacientes foram divididos em cinco grupos de números de ovos. A percentagem de pacientes que permaneceram no mesmo grupo no qual haviam sido enquadrados no primeiro exame foi $62,5 \%$; este indice foi estatisticamente semelhante entre os que eliminavam $<100,100-499,500-999,1000-2999$ ou $\geqslant 3000$ ovos de $S$. mansoni por grama de fezes.

Tabela 1 - Estabilidade na contagem de ovos de Schistosoma mansoni quando os pacientes foram divididos em cinco classes de numeros de ovos; comparação entre os resultados

do primeiro exame e a média obtida em três exames de fezes

\begin{tabular}{|c|c|c|c|c|c|}
\hline \multirow{2}{*}{$\begin{array}{l}\text { Conta- } \\
\text { gem de ovos } \\
\text { no primeiro } \\
\text { exame (numero } \\
\text { de ovos por } \\
\text { grama de fezes) }\end{array}$} & \multirow{2}{*}{$\begin{array}{l}\text { Média do } \\
\text { número de } \\
\text { ovos em três } \\
\text { exames }\end{array}$} & \multicolumn{2}{|c|}{ Pacientes que mudaram de grupo } & \multirow{2}{*}{$\begin{array}{c}\text { Pacientes que } \\
\text { permaneceram } \\
\text { no mesmo grupo } \\
\text { (n) } \%\end{array}$} & \multirow{2}{*}{$\begin{array}{l}\text { Total } \\
\text { (n) } \%\end{array}$} \\
\hline & & $\begin{array}{l}\text { Para o grupo ime- } \\
\text { diatamente supe- } \\
\text { rior ou inferior } \\
\text { (n) } \%\end{array}$ & $\begin{array}{l}\text { Para outro } \\
\text { grupo } \\
\text { (n) } \%\end{array}$ & & \\
\hline$<100$ & & (10) 34.5 & (0) 0,0 & (19) 65,5 & (29) 100,0 \\
\hline $100-499$ & & ( 6 ) 21,4 & (1) 3,6 & (21) 75,0 & (28) 100,0 \\
\hline $500-999$ & & (11) 37,9 & (0) 0,0 & (18) 62,1 & (29) 100,0 \\
\hline $1000-2999$ & & (11) 37,9 & (0) 0,0 & (18) 62,1 & (29) 100,0 \\
\hline$\geqslant 3000$ & & (14) 48.3 & (1) 3,4 & (14) 48,3 & (29) 100,0 \\
\hline Total & & (52) 36,1 & (2) 1,4 & (90) 62,5 & (144) 100,0 \\
\hline
\end{tabular}

$\mathrm{X}^{2}=7,58$ (nào significante)

$O$ indice de estabilidade na contagem de ovos foi 72,9 e $81,2 \%$ quando os pacientes foram divididos, respectivamente, em quatro (classificação A) e em três (classificação $\mathrm{B} \mathrm{e} \mathrm{C}$ ) grupos de números de ovos (a diferença entre estes dois indices não foi estatisticamente significante; $\left.\mathrm{X}^{2}=3,24\right)$. $\mathrm{Na}$ classificação A, o indice de estabilidade foi semelhante nos quatro grupos analisados; na classificação $\mathrm{B}$, este indice foi maior nos pacientes que apresentavam $<500$ ovos/g fezes; na classificação C, o indice de estabilidade foi maior nos pacientes que apresentavam $<500$ $\mathrm{e} \geqslant 1000$ ovos/g fezes (Tabela 2).

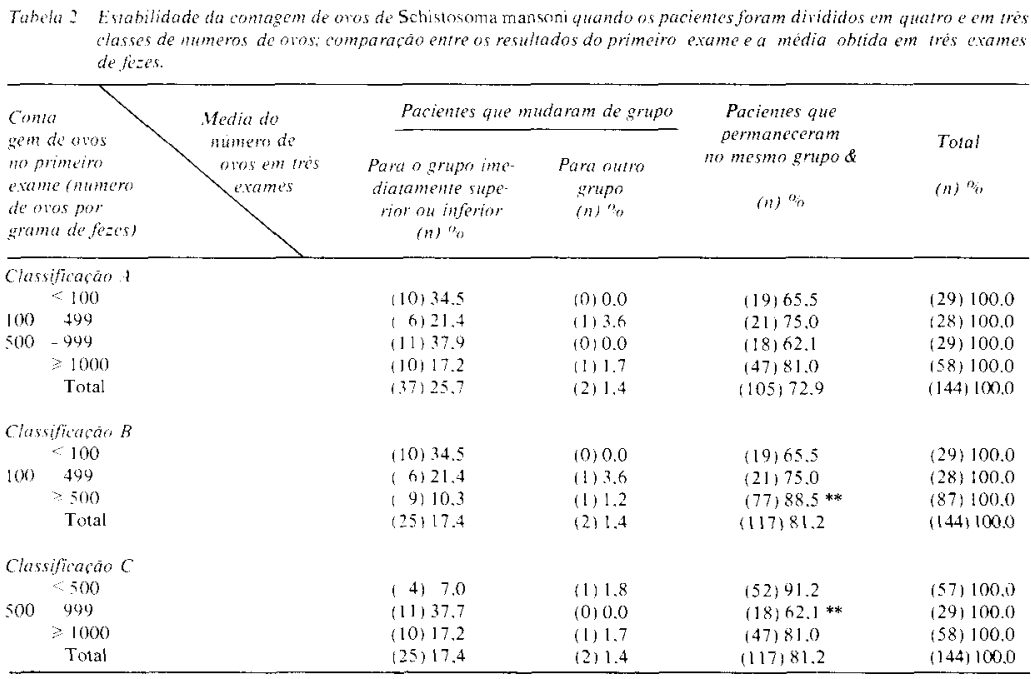

$x_{A}^{2}-7.23(N S): X_{B}^{2}=11.18(p<0.05): X_{C}^{2}=11.64(p<0.01)$

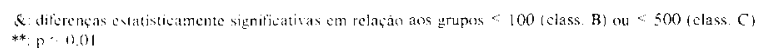


Lima e Costa MFF, Rocha RS, Katz N. Avaliaçāo da estabilidade na contagem de ovos de Schistosoma mansoni pelo método de Kato-Katz em uma zona endêmica da esquistossomose. Revista da Sociedade Brasileira de Medicina Tropical 17: 7-12, JanMar, 1984

É importante salientar que o coeficiente de correlação de Pearson entre o primeiro e o resultado obtido nos três exames de fezes foi alto $(r=0,9380)$ e estatisticamente significativo (Figura 1). A media geométrica do número de ovos de $S$. mansoni obtida no primeiro exame $(575,7 \pm 5,4)$ foi semelhante à obtida em três exames de fezes $(556,6 \pm 4,4 \mathrm{ovos} / \mathrm{g}$ fezes) $(t=0,19 ;$ não significante $)$.

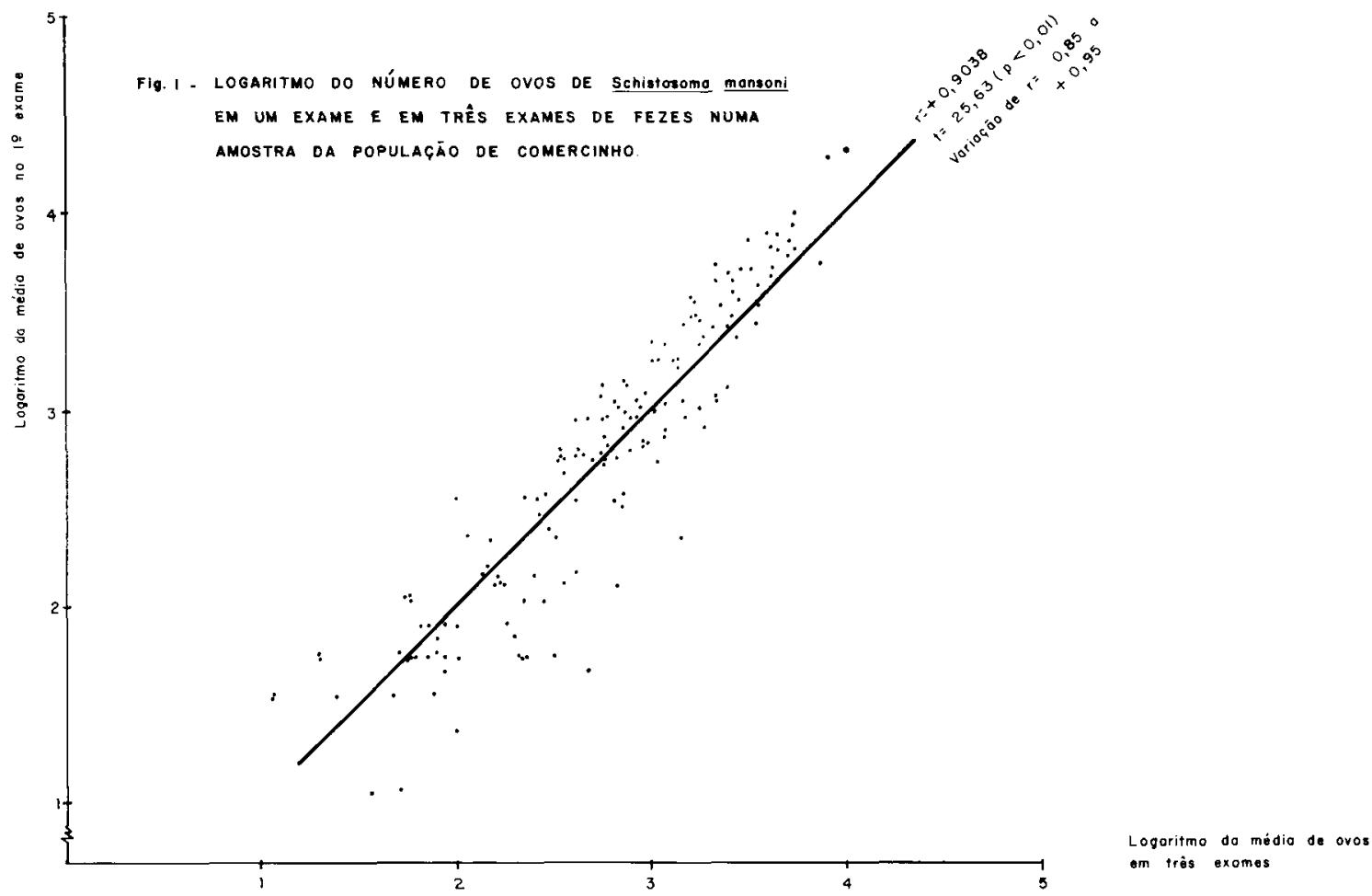

\section{DISCUSSÃO}

Diversos autores estudaram a estabilidade da contagem de ovos de $S$. mansoni pelo método de Kato em áreas endêmicas da esquistossomose. Katz e Zicker ${ }^{10}$ examinaram durante três dias consecutivos cerca de 90 pessoas residentes em Tuparecê, no interior de Minas Gerais, verificando que a mediana do número de ovos obtida no primeiro exame era semelhante à obtida no segundo e no terceiro exames de fezes. Siongok e cols ${ }^{13}$ repetiram, após três semanas, o exame de fezes pelo método de Kato em $10 \%$ da população de um área endêmica do Quênia, observando que $68 \%$ dos pacientes permaneciam no mesmo grupo $(<100,101-400,401-999$ e $\geqslant 1000 \mathrm{ovos} / \mathrm{g}$ fezes) no qual haviam sido enquadrados quando foi realizado o primeiro exame de fezes na localidade. Resultados semelhantes foram obtidos por Smith e $\operatorname{cols}^{14}$, também no Quênia, quando repetiram após 12 semanas o exame de fezes pelo método de Kato em $20 \%$ da população de uma pequena localidade. Bar- reto e cols ${ }^{2}$ fizeram exames de fezes durante três meses consecutivos em 23 pacientes que residiam numa zona endèmica da esquistossomose no Estado da Bahia. O logaritmo da média de número de ovos de $S$. mansoni nas fezes foi semelhante em cada um dos meses em que os exames foram efetuados e o coeficiente de correlação de Pearson foi alto para todos os períodos analisados. Lambertucci e cols $^{12}$ fi ?eram exames de fezes pelo metodo de Kato-Katz ( $\mathrm{m}$ dois dias consecutivos durante 10 meses) em 38 crianças residentes em Belo Horizonte. Os pacientes foram classificados em três grupos $(<100,100-499 \mathrm{e} \geqslant 500$ ovos/g), de acordo com a contagem de ovos obtida no primeiro exame. Após 10 meses verificaram que somente 11 pacientes permaneceram durante todo $o$ tempo no grupo inicial.

Domingues e cols ${ }^{5}$ observaram, em pacientes hospitalizados, que a estabilidade da contagem de ovos de S. mansoni pelo método de Kato-Katz aumentava consideravelmente quando os pacientes 
Lima e Costa MFF, Rocha RS, Katz N. Avaliação da estabilidade na contagem de ovos de Schistosoma mansoni pelo método de Kato-Katz em uma zona endémica da esquistossomose. Revista da Sociedade Brasileira de Medicina Tropical 17: 7-12, JanMar, 1984

eram submetidos a dieta padronizada, a tratamento prévio de helmintíases e protozooses intestinais, quando não recebiam medicamento que pudesse alterar a cor de suas fezes e quando o exame era feito na ausência da melena e/ou diarréia. Em estudos de campo, as condições avaliadas por estes autores, e mesmo a repetição do exame de fezes na população de uma zona endêmica, são extremamente dificeis de serem reproduzidas.

O presente trabalho foi desenvolvido com o objetivo de avaliar, numa área endèmica, em que medida as contagens de ovos de $S$. mansoni obtidas em um e em três exames de fezes pelo método de KatoKatz se correspondem. Os resultados deste estudo mostraram que as médias geométricas do número de ovos de $S$. mansoni obtidas em um e em três exames de fezes foram semelhantes. Isto confirma as observações de Katz e cols ${ }^{11}$ e de Barreto e cols ${ }^{2}$ de que as contagens médias de ovos do parasita, obtidas em grupos de pacientes, apresentam alto grau de estabilidade. Quando os pacientes foram analisados individualmente, a estabilidade na contagem de ovos variou entre 62,5 e $81,2 \%$, dependendo do número de classes nas quais os pacientes foram divididos (Tabelas 1 e 2); estes indices foram razoavelmente semelhantes aos obtidos em duas zonas endêmicas do Quênia $(68-70 \%)^{1314}$

Diante destes resultados pode-se concluir que: a) a contagem de ovos de $S$. mansoni, expressa em média geométrica, apresentou alto grau de estabilidade, sendo que os resultados obtidos em um e em très exames de fezes se corresponderam; b) a percentagem de pacientes que permaneceram na mesma classe de número de ovos na qual haviam sido enquadrados no primeiro exame foi estatisticamente semelhante nas classificações A, B e C (72,9-81,2\%); c) no estágio atual de conhecimentos, a opção por uma destas três classificações dependerá dos objetivos do estudo e das características das zonas endêmicas a serem investigadas; a classificação $\mathrm{C}$, por exemplo, pode ser adotada nas áreas onde existam grande número de pacientes com mais de 1000 ovos de $S$. mansoni por grama de fezes e/ou nas áreas onde serão estudados comparativamente pacientes com altas e baixas contagens de ovos do parasita, tendo em vista que nesta classificação os extremos apresentaram maiores indices de estabilidade. De qualquer forma, uma classificação definitiva para o método de Kato-Katz só será possível qüando as relações entre a contagem de ovos de $S$. mansoni nas fezes e a morbidade da esquistossomose estiverem bem definidas.

\section{SUMMARY}

The stability of S. mansoni egg count, using Kato-Katz method, was evaluated in 144 persons living in an endemic area of Minas Gerais State. The results obtained with the first stool examination were compared to the mean obtained with three examinations performed within a period of eleven weeks. The patients were classified in three different groups according to the number of eggs found in the first stool examination: $<100,100-499,500-999$ and $\geqslant 1000$ eggs (classification A); < 100, 100-499 and $\geqslant 500$ eggs (classification B); < 500, 500-999 and $\geqslant 1000$ eggs (classification $C$ ). The proportion of patients that remained within the same class of number of eggs (73-81\%) was statistically similar in classifications $A, B$ and $C$. The Pearson correlation coeficient was high ( $r=0,9038)$ and the geometric mean number of eggs with the fist examination was similar to the one obtained with the three stool examinations $(575.7 \pm 5.4$ and $556.6 \pm 4.4$, respectively).

Keywords: Schistosomiasis mansoni. Schistosoma mansoni. Stool examination. Kato-Katz method.

\section{AGRADECIMENTOS}

Os autores agradecem aos senhores Gercy Souza Morais, Pedro Coura e José Ribeiro, da equipe do Laboratório de Esquistossomose do Centro de Pesquisas "René Rachou" - FIOCRUZ.

\section{REFERÊNCIAS BIBLIOGRÁFICAS}

1. Abdel-Wahab MF, Strickland GT, El Sahly A, Ahmed L, Zakarias S, El Kady N, Mahmoud S. Schistosomiasis mansoni in an Egyptian village in the Nile Delta. American Journal of Tropical Medicine and Hygiene 29:868-864, 1980.

2. Barreto ML, Silva JTF, Mott KE, Lehman JSJr. Stability of faecal egg excretion in Schistosoma mansoni infection. Transactions of the Royal Society of Tropical Medicine and Hygiene 72:181-187, 1978.

3. Conceição MJ. Morbidade da esquistossomose mansoni em uma comunidade rural de Minas Gerais. Tese de mestrado, Universidade Federal do Rio de Janeiro, Rio de Janeiro, 1976.

4. Costa MFFL. Estudo clínico-epidemiológico da esquistossomose em Comercinho, Minas Gerais (1974/1981) Tese de doutorado, Universidade Federal de Minas Gerais, Belo Horizonte, 1983. 
Lima e Costa MFF, Rocha RS, Katz N. Avaliação da estabilidade na contagem de ovos de Schistosoma mansoni pelo método de Kato-Katz em uma zona endémica da esquistossomose. Revista da Sociedade Brasileira de Medicina Tropical 17: 7-12, JanMar, 1984

5. Domingues L, Silveira M, Vanderlei MI, Kelner S. Possiveis fatores que alteram os resultados da coproscopia quantitativa de ovos de $S$. mansoni pelo método de Kato-Katz. Revista do Instituto de Medicina Tropical de São Paulo 22:1 14-117, 1980.

6. Guimarães MDC. A schistosomiasis mansoni clinicalepidemiologic study in a small Brazilian community. Thesis of master, Hebrew University, Jerusalém, 1982.

7. Hiatt RS. Morbidity from Schistosoma mansoni infections: an epidemiologic study based on quantitative analysis of egg excretion in two highland Ethiopian villages. American Journal of Tropical Medicine and Hygiene 25: 808-817, 1976.

8. Hiatt RS. Gebrin-Medhin M. Morbidity from Schistosoma mansoni infection: an epidemiologic study based on quantitative analysis of egg excretion in Ethiopian children. American Journal of Tropical Medicine and Hygiene 26: 473-481, 1977.

9. Katz N, Chaves A, Pellegrino J. A simple device for quantitative stool thick-smear technique in schistosomiasis mansoni. Revista do Instituto de Medicina Tropical de São Paulo 14: 397-400, 1972.

10. Katz N, Zicker F. Correlation between symptomatology and intensity of Schistosoma mansoni infection in inhabitants from endemic areas in Minas Gerais stateBrazil. Brasilia Medica 11: 55-49, 1975.

11. Katz N, Zicker F, Rocha RS, Oliveira VB. Re-infection of patients in schistosomiasis mansoni endemic areas after specific treatment: I influence of age and worm burden. Revista do Instituto de Medicina Tropical de São Paulo 20: 273-278, 1978.

12. Lambertucci JR, Pedroso ERP, Greco DB, Rocha MOC. Estabilidade na excreção de ovos de $S$. mansoni nas fezes de crianças com esquistossomose mansônica crônica. Revista do Instituto de Medicina Tropical de São Paulo 25: 67-72, 1983.

13. Siongok TKA, Mahmoud AAF, Ouma JH, Müller AS, Handa AK, Houser HB. Morbidity in schistosomiasis mansoni in relation to intensity of infection: study of a comunity in Machakos, Kenya. American Journal of Tropical Medicine and Hygiene 25: 273-284, 1976.

14. Smith DH, Warren KS, Mahmoud AAF. Morbidity of schistosomiasis mansoni in relation to intensity of infection; study of a community in Kisumi, Kenya. American Journal of Tropical Medicine and Hygiene 28: 220-229, 1979.

15. Spiegel MR Estatística, 10a edição. Editora Mc GrawHill do Brasil Ltda, Sào Paulo, 1976. 